\title{
Multiple Referrals and Multidimensional Cheap Talk
}

\author{
Marco Battaglini ${ }^{\infty}$ \\ J anuary 31, 2000
}

\begin{abstract}
A bstract
Cheap talk games have been widely used to analyze situations in which a policy maker needs expert advice. In previous work, agent uncertainty has almost always been modeled using a single-dimensional state variable. In this paper we prove that the dimensionality of the uncertain variable has an important qualitative impact on results and yields interesting insights into the $\backslash$ mechanics" of information transmission. Contrary to the unidimensional case, with more than one dimension full transmission of information in all states of nature is typically possible, provided a very simple and intuitive condition is satis ${ }^{-}$ed. What really matters in transmission of information is the local behavior senders' indi Rerence curves at the ideal point of the policy maker (receiver), not the proximity of players' ideal point. This may explain the apparent con ${ }^{\circ}$ ict between informational theories of committees and the empirical evidence on the poor alignment of preferences between the Floor and committees in legislative organizations.
\end{abstract}

\footnotetext{
${ }^{\circledR}$ E conomics Department, Northwestern University, 2003 Sheridan Road, Evanston, Illinois 602082600. e-mail: m-battaglini@nwu.edu. I am grateful to David Austen-Smith, A riel Burstein, Matthew J ackson, Hugo Nopo, Wolfgang Pesendorfer, J oel Sobel, Sandy Zabell and the participants of the $\backslash$ Wallis Conference on Political E conomy" (Rochester, NY, October 1999) for helpful comments. I am especially indebted with Tim Feddersen and A sher Wolinsky for advice and encouragement. All remaining errors are mine. Financial support from Banca San Paolo-IMI is gratefully acknowledged.
} 


\section{Introduction}

In 1977, when the United States House of Representatives discussed the \Clean A ir A ct", two issues were at stake: on the one hand the impact of the act on the economy (through, for example, increased unemployment); on the other hand the act's environmental and health bene ${ }^{-}$ts. The optimal decision, clearly, had to deal with this trade-o ${ }^{\circledR}$ and so had to be conditioned on inside information regarding the likely eßects. As A usten-Smith [1990] notes, the lobbies (the auto industry on one side; environmentalists on the other) had much better information than the House, but, also, had strong biases along one dimension or the other. Despite the con ${ }^{\circ}$ icts of interest with the policy maker, their strategic interaction resulted in the transmission of a good deal of information. The । interested experts", however, selected strategically the data which they disclosed:

Proponents of tightening regulation on emissions and so forth presented a host of information on the health and environmental consequences of the bill, they almost wholly ignored the focus of their opponents who in turn argued against for the regulation almost exclusively on economic grounds (especially rising unemployment in the auto industry)(Austen-Smith [1990], p. 408)

The outcome of this \expertise game" was the result of the strategic interaction of two competing agents with a con ${ }^{\circ}$ ict of interest along di ßerent dimensions of the same problem. This is an example of a whole class of situations (probably all) in which the policy decision is multidimensional. Understanding these situations seems important not only for positive analysis: it is a prerequisite for the optimal design of legislative or private organizations. This is not a novelty: the literature has paid careful attention to the organizational implications of information transmission in legislative games ${ }^{1}$. Yet, despite its importance, with the notable exception of A usten-Smith [1990]2, almost no work on information transmission in legislative games has analyzed the implications of the multidimensionality on the problem; typically, in fact, in cheap talk games the relevant choice to be made is a point in the real line ${ }^{3}$. Clearly the unidimensional assumption is not justi ${ }^{-}$ed because it is realistic: but it might be appropriate as a $\backslash^{\text {" }}$ rst order" approximation if it does not have a qualitative impact on the results. In this case these models might be seen as \reduced forms" of a more complex environment: for example a model in which the policy space is multidimensional but the policy makers have only one

\footnotetext{
${ }^{1}$ See Gilligan and Krehbiel [1989] and A usten Smith [1991]; for an extensive survey of the topic see K rehbiel [1991].

${ }^{2}$ Discussion of Austen-Smith's results will follow.

${ }^{3}$ T his is not a limit only of the literature on information transmission applied to legislative games: no general theory of cheap talk in multidimensional environment exists.
} 
dimensional jurisdictions. However, the generality of some results of these works become questionable if the dimensionality of the problem changes qualitatively the results.

This paper has a main message to convey: the analysis of an environment with more than one dimension is not just a technical change but is qualitatively di ßerent and yields interesting insights into the study of the etciency of information transmission in the policy making process. In order to prove this conjecture, the analysis in this paper will follow two logical steps.

First, we will revisit the unidimensional analysis. As we mentioned, all the existing work on cheap talk games ${ }^{4}$ is in one dimension. Crawford and Sobel [1982] began this literature analyzing the case of one informed agent (sender) and an informed principal (receiver) who makes a decision based on the message of the sender; they prove that no fully revealing equilibrium exists and characterize a particularly important class of equilibria ${ }^{5}$. Only recently, however, the two-sender case has been analyzed in the pioneering works of Gibbons [1988], Gilligan and K rehbiel [1989], A usten Smith [1991]; and the more recent works of Epstein [1997], K rishna and Morgan [1999a] and Krishna and Morgan [1999b]. Although these works di ßer in modelling choices and assumptions, they have a common trait in line with the result of Crawford and Sobel [1982]: full revelation of information is not typically achieved even with multiple referrals and the informativeness of the equilibria is positively correlated with the proximity of the ideal points of the experts and the policy maker. However conditions for the non-existence of a fully revealing equilibrium are not completely characterized ${ }^{6}$. In the ${ }^{-}$rst section of the paper, we complete the analyses of the previous papers by showing that even if full revelation is possible in some cases, these outcomes are supported by equilibria that are not plausible since they critically rely on an ad hoc construction of out-of-equilibrium beliefs and do

\footnotetext{
${ }^{4} \mathrm{~A}$ cheap talk game is a signalling game in which neither the sender's nor the receiver's payo ${ }^{\circ}$ depends on the messages sent: payo ${ }^{\circledR S}$ are only functions of types and the action taken by the receiver.

${ }^{5}$ Partitional equilibria: they prove that for any perfect bayesian equilibrium there exists a payo ${ }^{\circledR}$ equivalent partitional equililibrium.

${ }^{6} \mathrm{~K}$ rishna and Morgan [1999a] prove that when experts report sequentially no fully revealing equilibrium exists; but Gilligan and K rehbiel, who ${ }^{-}$rst have considered the simultaneous report case, do not prove that when experts report simultaneously no fully revealing equilibrium exists. In eRect, just a slight generalization of the model employed by Gilligan and K rehbiel may yield the existence of fully revealing equilibria. This is true when experts have like biases, as shown by K rishna and M organ [1999a], but also it might be true, under some conditions, in the case with opposed biases of the experts with respect to the policy maker. In the ${ }^{-}$rst section we present a necessary and su \pm cient condition for the non existence of a fully revealing equilibrium even in the cases that have not been analyzed before. K rishna and $M$ organ [1999b] have, independently from this work, found a su \pm cient condition for the existence of a f.r.e.

The modelling approach of Austen Smith is rather di ßerent and so not directly comparable with the papers by Gilligan and K rehbiel or K rishna and M organ. In A usten Smith's paper experts do not observe the true state but a sample of drawings from a known distribution of it. In this approach, more than the concept of fully revealing equilibrium is relevant the concept of $\backslash$ coherence" of the equilibrium.
} 
not survive a simple re- nement. The conclusion that we draw from this section is that in one dimension there is no equilibrium with good properties in which information is fully revealed.

Given this, we proceed to the multidimensional problem. The main result of this section and of the paper is that if there are 2 dimensions and 2 experts with a mild $\operatorname{con}^{\circ}$ ict of interest, then there exists a fully revealing equilibrium that survives the above intuitive re nements: under the assumption of quadratic utilities, we require ideal points to be linearly independent ${ }^{7}$. The result is interesting because it is essentially qualitative: if ideal points are \just an " linearly independent (for example if $x_{i}$ and $x_{j}$ are the ideal points of the experts, then $x_{i}={ }^{B} x_{j}+$ ") then the result holds; therefore the nonexistence of fully revealing equilibria is not a \ continuous" result in the dimensionality of the problem. The result is robust to changes in a) the information structure, since, even if experts do not know the ideal point of the other expert, the equilibrium exists; $b$ ) timing of referrals, since even if agents report sequentially, provided a very simple and intuitive condition on the direction of the highest increase in the experts' utility at the policy maker's ideal point is satis ${ }^{-}$ed, the equilibrium exists. Even more than the existence result, we believe that the analysis yields important insights into the \mechanics" of transmission of information that are true also in more general frameworks:

1. Importance of the local behavior of the experts' indi ßerence curves at the ideal point of the policy maker. As we said, a typical result of unidimensional cheap talk games is that the closer the ideal points, the more information transmitted in equilibrium ${ }^{8}$. However, if the informational theory of legislative organizations is to be accepted, there is a substantial empirical puzzle. As Londregan and Snyder[1994] put it:

The dominant view among congressional scholars is that many congressional committees and sub committees are not representative of the entire chamber from which they are selected but instead have a relatively strong preference for serving particular interests (Londregan and Snyder [1994], p.233)

Although formal empirical analyses have presented mixed results, even the empirical studies that support more the informational theory, show signi ${ }^{-}$cant violation of the $\backslash$ out-

\footnotetext{
${ }^{7} \mathrm{~W}$ hen the outcome space is $<^{\mathrm{d}}$, and utilities are strictly concave, an ideal point is a well de- ned vector in $<$ : If we normalize the policy maker's ideal point at zero, linear independence means that experts ideal point can not be expressed as $x_{1}={ }^{\circledR} X_{2}$ for ${ }^{\circledR} 2<$ : See below for details.

${ }^{8} \mathrm{~K}$ rehbiel states it as one of the $\backslash$ " ve principles of legislative signalling" :
}

THE OUTLIER PRINCIPLE. The more extreme are the preferences of a committee specialist relative to preferences of a non specialist in the legislature, the less informative is the committee ...the outlier principle is the most robust property of legislative signalling models. (K rehbiel [1991], p 81) 
lier principle". For example, although $\mathrm{K}$ rehbiel [1991] ' $\mathrm{nds}$ that many committees have no signi ${ }^{-}$cant ideological bias relative to the ${ }^{\circ}$ oor $^{9}$, he also ${ }^{-}$nds that one third of the committees has statistically signi ${ }^{-}$cant ideological bias ${ }^{10}$ : Foreign $\mathrm{A}$ Rairs, Education and Labor, Post $\mathrm{O} \pm$ ce and Civil Service, Armed Services, District of Columbia. The results of the empirical work of Londregan and Snyder [1994] are pretty drastic:

These results are inconsistent with the implications of models that emphasize the asymmetric information problems arising from committee expertise... (Londregan and Snyder [1994], p.262)

Very little work has been done to reconcile theory with empirical evidence. The only theoretical solution to this puzzle has been provided by the theory of congressional hearings by Diermeier and Feddersen [1998]: the authors prove that even when the ideal points of the experts are not close to the policy maker's, there may be transmission of information. Hearings, in fact, under very plausible assumptions ${ }^{11}$ work as signalling devices for commissions (experts) and allow transfers of information.

In this paper, we show that when the analysis is multi-dimensional, the evidence described above is not at odds with an informational theory of legislative organizations. In a multidimensional setting, we prove that even experts with extreme preferences may provide useful information, even if costly signalling is not allowed ${ }^{12}$. What really matters for the existence of a fully revealing equilibrium in this case is the local behavior of experts' utilities at the ideal point of the policy maker. If utilities are di ßerentiable, the gradient of utilities at the policy maker's ideal point is crucial to existence, a result that has the ${ }^{\circ}$ avor of the existence results in the social choice literature ${ }^{13}$. The theory presented therefore questions the $\backslash$ outlier principle" as a characterizing feature of legislative signalling models, but provides strong support for the informational theory of legislative organizations.

2. O pen rule vs. closed rule. The multi-dimensional case yields useful insights into the analysis of the Iopen rule" vs. Iclosed rule" question, a classic topic of analysis in the legislative cheap talk games (see Gilligan and Krehbiel 1987, 1989, 1990). In the one-dimensional setting, the meaning of \closed rule" is obvious: having a \closed rule" power in one dimension means that the policy maker is constrained to choose between the proposal and the status quo; there is no question about in which dimension power is granted. In the multi-dimensional case considered in this paper, however, dimensions are

\footnotetext{
${ }^{9} \mathrm{~K}$ rehbiel [1989] uses uses A mericans for D emocratic Action ans A merican Conservative Union ratings.

${ }^{10}$ See K rehbiel [1989] p.128.

${ }^{11} \mathrm{Hearings}$ must be costly and somewhat informative.

${ }^{12}$ Since hearings are costly the model of Diertmeier and Feddersen is not a cheap talk model but a signalling model.

${ }^{13}$ See for example P lott [1967] or A usten Smith and Banks for an extensive survey [1998].
} 
endogenous ${ }^{14}$. W ith some degree of approximation, we may imagine a two stage process: - rst the policy maker decides the jurisdictions on which the expert is granted a closed rule proposal power, then the expert makes the proposal. This has interesting empirical implications: in equilibrium, we may observe that all the proposals of the expert are accepted by the policy maker, and this may be interpreted as evidence that the expert has a dominant position. However, when the ex ante choice is considered the interpretation may be inverted: the policy maker may have chosen optimally the dimension over which the expert reports in order to exploit his con ${ }^{\circ}$ ict of interest with other experts; the choice of the jurisdiction may be, as we will see, such that the optimal choice of the expert coincides with that of the policy maker. So the policy maker is really in a dominant position.

3. Separation of power and allocation of jurisdictions. More generally, to the extent that we may divide the policy space along dimensions, this approach allows us to discuss the issue of the allocation of jurisdictions and decision power. A government, in fact, is a set of agents and interacting rules designed to take decisions in multi-dimensional policy spaces ${ }^{15}$; similarly, a committee in the Congress is not a single agent, but a set of agents with a collective internal organization. The importance of the organization is strictly related to the multidimensionality of the policy space. Tasks inside the policy maker's organization are allocated as jurisdictions on subsets of the policy space dimensions: with only one dimension it is di \pm cult to imagine the meaning of $\backslash$ jurisdictions". The natural question is then how much jurisdictions matter in the decision process. In some sense the analysis here parallels the approach that regards the ${ }^{-} \mathrm{rm}$ as an organization and not simply as a black box. In the presence of multiple equilibria the ex ante allocation of jurisdictions may have important $\backslash$ focal" eßects on the selection of the "nal outcome.

\subsection{Related literature}

The importance of multidimensionality in spatial models of voting has been recognized at least since the sixties with extensions of the Hotelling model by Davis and Hinich [1966] and the equilibrium conditions for existence discovered by Plott [1967] ${ }^{16}$. However in models of legislative organization and in cheap talk games in general, as we said, very little work has been done. Austen-Smith [1993], actually, is the only work that addresses the problem. Austen-Smith has a modelling approach that is completely di ßerent from the one described here and employed in other papers (Gilligan and K rehbiel [1987], Epstein

\footnotetext{
${ }^{14}$ We will be more precise on this later, for now note that one point in $<^{2}$ is represented by the two coordinates of some coordinate system, each coordinate is a dimension of the policy space

${ }^{15}$ See Laver and Shepsle [1994] on this.

${ }^{16}$ See also A usten-Smith and B anks [1998] for an excellent survey.
} 
[1998], K rishna and M organ [1999]): experts do not observe the true state of the world but a random sample from a known distribution. This approach is more realistic but more di \pm cult to analyze. In one dimension, it yields very elegantly clear and general results, but in more than one dimension becomes exceedingly di \pm cult to analyze: therefore in the multidimensional case it has not been possible to characterize general results, but only to present some examples. The examples of Austen-Smith, however, yield very interesting intuitions that are completely in line with the results of this paper. Although we do not study the choice of specialization in information production (as A usten-Smith does), we con $^{-} \mathrm{rm}$ A usten Smith intuition that experts will be in ${ }^{\circ}$ uential only on some dimension of the policy space and therefore will argue on \orthogonal issues".

A nother work on information transmission in a multidimensional setting is Milgrom and Roberts [1986]. There are two main di ßerences between it and our work. First, Milgrom and Roberts assume that information is veri ${ }^{-}$able: experts may conceal information, but they can not lie. Second, we prove that thanks to multidimensionality we can achieve full revelation; they prove that despite multidimensionality (and if information is veri' ${ }^{-}$able), we may ${ }^{-}$nd a fully revealing equilibrium. In this sense, multidimensionality makes the disclosure game more complicated, but it does not change it qualitatively.

This paper is also related to Farrell and Gibbons [1989]. In our model each declarations by an Expert has an externality on the relationship between the other Expert and the Receiver. In particular, the set on which the Expert is in ${ }^{\circ}$ uential is conditional on the other Expert's message. The multidimensionality of the problem helps to best exploit these externalities. In Farrell and Gibbons, there is only one Expert but two Receivers: the declaration of the Expert to one Receiver has an externality on the relationship that the Expert has with the other Receiver. The authors prove that these externalities may be exploited to extract more informative signals from the Sender.

\section{The model}

Policy space, players, preferences. Let $Y^{\prime}<^{d}$ denote the set of alternatives for the policy maker. Following A usten Smith and Riker (1987), we distinguish between the policy space and the outcome space. Policy choices do not coincide with outcomes. For any decision of the policy maker ( $y 2 Y$ ), the outcome is $x=y+\mu$ where $\mu$ is a d-dimensional vector. Nature chooses $\mu$ according to some continuous distribution function $F(\mu)$ with density $f(\mu)$; support $f$ and zero expected value. The most reasonable assumption is that the domain of $\mu$ is not restricted a priori : although, in fact, extreme states may occur with negligible probability, we do not want to rule them out. So, if not otherwise speci ${ }^{-}$ed, we assume that $f^{\prime}<$. Consider Fig.1: if the state is $\mu$ and the policy maker chooses $y$, 


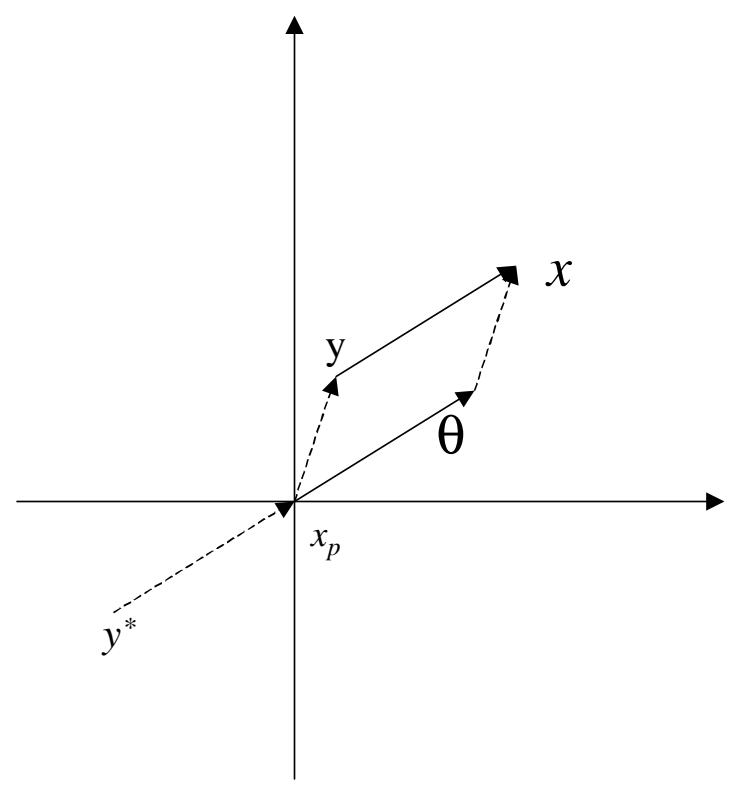

Figure 1: If the state is $\mu$ and the policy maker chooses $y$, the outcome is $x$ : W ith simmetric information the optimal choice for the policy maker is $y^{a}=i \mu$ :

the outcome is $x$. With symmetric information, the optimal choice for the policy maker, if the ideal point is the origin, is $\mathrm{y}^{\not}=\mathrm{i} \mu$. As in A usten Smith and Riker (1987) and Gilligan and K rehbiel (1989) the policy maker chooses y without knowledge of $\mu$. The experts instead observe the realization of nature. This is the central asymmetric feature of the model.

There are three players and each of them has Von Neumann M orgenstern utility function $u_{i}: X !<$ We assume that the $u_{i} s$ are continuous, strictly concave, di ßerentiable. The ${ }^{-}$rst two agents are called experts (the set of experts is $E$ ), each expert has an ideal point $x_{i}$. The policy maker has ideal point $x_{p}$ that we normalize to be at the origin, i.e. $x_{p}=i_{0} 2<$ : For simplicity we will assume quadratic utilities, but this assumption is not relevant for the results and will be relaxed later: $u_{i}=i{ }_{j=1}^{d} x_{i}^{j} x^{j}{ }^{\phi_{2}}$ where $x_{i}^{j}$ is the $j$ coordinate of $i$ 's ideal point: Utility functions (and therefore ideal points) are common knowledge.

Timing. The timing of the interaction is as follows: a) at time 0 nature chooses $\mu$ according to $F(\mu)$ and each Expert observes the true $\mu ; b)$ at time 1 the Experts are asked to report simultaneously or privately the state of nature $\mu$ to the policy maker; $c$ ) the Policy Maker decides $y$ and the outcome that is realized is $x=y+\mu$.

Strategies and equilibrium. A strategy for the policy maker is a function $y: f f f ! Y$, 
i.e. for each couple of declarations of the experts associates an element of $Y^{17}$. A strategy for the ith expert is a function $s_{i}: £ ! f$, for each realization of nature the expert reports $\mu 2 f$. A belief function for the policy maker is a function ${ }^{1}: f f f ! P(f)^{18}$ : for each pair of proposals of the experts assesses a posterior probability distribution over $f$. An equilibrium is de ${ }^{-}$ned as follows:

De $e^{-}$nition 1 An equilibrium in d dimensions with 2 experts is a collection of strategies $y\left(s_{1} ; s_{2}\right) ; s_{i}(\mu)$ 8i $2 f 1 ; 2$ gand a belief ${ }^{1}\left(s_{1} ; s_{2}\right)$ such that:

a) $8 \mathrm{i} 2 \mathrm{f} 1 ; 2 \mathrm{~g} \mathrm{~s}_{\mathrm{i}}(\mu)$ maximizes $E u_{i}$ given $y\left(s_{1} ; s_{2}\right) ; s_{i} i(\mu)$;

b) $y\left(s_{1} ; s_{2}\right)$ maximizes $E u_{p}$ given $s_{i}(\mu) 8 i 2 f 1 ; 2 g$ and ${ }^{1}\left(s_{1} ; s_{2}\right)$

c) ${ }^{1}\left(s_{1} ; s_{2}\right)(\mu)$ is formed using experts' strategies $s_{i}(\mu)$ by using $B$ ayes' rule whenever possible.

A fully revealing equilibrium is an equilibrium in which for each true state $\mu,{ }^{1 x}\left(S_{1}^{\mathrm{a}}(\mu) ; \mathrm{S}_{2}^{\mathrm{a}}(\mu)\right)(\mu)=$ 1: i.e. for any state of the world information is perfectly transmitted in equilibrium. Note that it is di \pm cult to achieve a fully revealing equilibrium because the policy maker's action must be sequentially rational. If the policy maker could commit to a policy response to the declarations of the experts, then it would be much easier to implement a fully revealing equilibrium with strategies of the type: \if the experts's reports disagree, the policy maker would choose a policy that is bad for everyone", this is generally possible but would not be sequentially rational.

It is useful to introduce a further de nition and a simple lemma. In a fully revealing equilibrium, as de- ned in the previous paragraph, the true state is al ways revealed to the policy maker: however this does not imply that in equilibrium experts report the truth, any function of the true state will do as well if the policy maker 'understands' it: this multiplicity of equilibria is a well known characteristic of cheap talk games. We de-ne a truthful fully revealing equilibrium a fully revealing equilibrium in which experts report what they observe truthfully.

Lemma 1 If there exists a fully revealing equilibrium then there exists a truthful fully revealing equilibrium. If the truthful fully revealing equilibrium has non degenerate out of equilibrium beliefs (i.e. beliefs that assign positive probability to more than one state of nature), then there exists a truthful fully revealing equilibrium with degenerate out of equilibrium beliefs, i.e. beliefs that assign probability to only one state of nature.

\footnotetext{
${ }^{17}$ Given the assumption of strict concavity of utilities, we might restrict attention to pure strategies without loss of generality.

${ }^{18} \mathrm{P}(\mathrm{f})$ is the set of probability distributions over $\mathrm{f}$.
} 
Proof. In the appendix.

This simple lemma is useful in proving the non existence of a fully revealing equilibrium: if we prove that no truthful fully revealing equilibrium exists than the lemma implies that no fully revealing equilibrium exists. The intuition of the lemma is very simple. The ${ }^{-}$rst part is similar to the revelation principle; in the second part (degenerate beliefs) we show that for any belief ${ }^{1}\left(s_{1}(\mu) ; s_{2}(\mu)\right)$ the optimal choice of the policy maker is $y\left(s_{1}(\mu) ; s_{2}(\mu)\right)=i E_{1}\left(s_{1}(\mu) ; s_{2}(\mu)\right)(\mu)$ : Then for any belief we construct a degenerate belief that assigns probability one to $E_{1}\left(s_{1}(\mu) ; s_{2}(\mu)\right)(\mu)$ : the equilibrium with these beliefs is outcome equivalent to the original one.

\section{R esults}

\subsection{Fully revealing equilibria in one dimension}

In this section we study the problem of fully extracting information from experts in a one dimensional setting. The goal of this section is to ${ }^{-}$nd conditions for the existence of fully reveling equilibria in order to compare the results with the case of higher dimensional policy spaces. The intention here is, more than to present previous work, to extend and unify its results in a general framework.

Gilligan and K rehbiel [1989] ' rst analyze a cheap talk model with multiple referrals, heterogeneous preferences and asymmetric information along one dimension. They ${ }^{-}$nd an equilibrium of the game that is not fully revealing and draw inferences on it, but they do not prove the non existence of a fully revealing equilibrium. However, as K rishna and Morgan [1999a] show, once we consider a slightly more general model, it is not obvious that no fully revealing equilibrium exists. Consider, for example, the case in which experts have like biases (i.e. ideal points are both larger (smaller) than the policy maker's): equilibria with full revelation always exist and, moreover, involve very simple strategies. Assume, for example, that $x_{i}>0$ for $i=1 ; 2$; consider these beliefs and strategies: ${ }^{1}\left(s_{1}(\mu) ; s_{2}(\mu)\right)\left(\max f s_{1}(\mu) ; s_{2}(\mu) g\right)=1 ; s_{i}(\mu)=\mu$ for $i=1 ; 2 ; y\left(s_{1}(\mu) ; s_{2}(\mu)\right)=$ i $\max _{\mathrm{f}} \mathrm{s}_{1}(\mu) ; \mathrm{s}_{2}(\mu) \mathrm{g}$ : Given the beliefs of the policy maker and $i$ 's strategy, $\mathrm{i} i$ 'nds optimal to reveal the truth. This equilibrium survives as a $P B E^{19}$. In a subsequent paper, independent from our research, K rishna and M organ [1999b] show that, in exactly the same model of Gilligan and K rehbiel [1989], if biases of the experts are very small, then there is a f.r.e.: however, they do not completely characterize the conditions in which a fully revealing equilibrium exists.

Full characterization of conditions for the existence of fully revealing equilibria in

\footnotetext{
${ }^{19}$ T his equilibrium, however does not survive if experts make mistakes with arbitrary small probability.
} 
one dimension is clearly important for the purposes of this paper, in order to put in perspective the results in higher dimensions: but it has also considerable interest in its own. Situations in which experts do not report sequentially seem the norm since policy makers do not generally disclose the advice of their experts. In this section therefore we make two points. In Proposition 1 we ${ }^{-}$nd a necessary and su \pm cient condition for the existence of a fully revealing equilibrium when experts report simultaneously and have opposed biases. In Propositions 2, however, we show that even if the conditions are violated, or if experts have like biases, no fully revealing equilibrium survives an intuitive re- nement.

The key to the existence of a fully revealing equilibrium in one dimension is $f$, i.e. the support of the variable $\mu$. The ${ }^{-}$rst result of this section is that when $f$ is large enough the policy maker can achieve a fully revealing equilibrium even if the agents have opposed biases. De $e^{-}$in this one-dimensional setting $f=[; W ; W]^{20}$ : The intuition behind the fact that fully revealing equilibria may exist is the following. Nothing prevents the policy maker from having out of equilibrium beliefs that are conditional on the observed messages. Notice that if this is the case, then deviations from a fully-revealing equilibrium become more di \pm cult because declarations reveal some information about the true state of world. When an expert contemplates a deviation from a fully revealing equilibrium, in fact, he must assume that the other expert and the policy maker follow the equilibrium strategies: therefore the expert knows that some information is revealed to the policy maker by the other expert even if he deviates. The larger $W$, the more freedom we have to ${ }^{-}$nd the function ${ }^{1}\left(s_{1} ; s_{2}\right)$ and so the larger is the set of equilibria.

$\mathrm{K}$ rishna and M organ [1999a] prove the non existence of a fully revealing equilibrium when experts report sequentially. In the following proposition we analyze the case of simultaneous reports and opposed biases (assume w.I.g. that $\mathrm{x}_{1}<0, \mathrm{x}_{2}>0$ ).

Proposition 1 If $d=1$ and the experts' ideal points $\left(\mathrm{x}_{1} ; \mathrm{x}_{2}\right)$ are on opposite sides of the policy maker's ideal point, then $j x_{1} j+j x_{2} j>W$ is a necessary and su \pm cient condition for the non existence of a fully revealing equilibrium

Proof. In the appendix

In proving Proposition 1 in the appendix, we show that if $W, j x_{1} j+j x_{2} j$ there is an entire class of equilibria that would be fully revealing; an example is the following:

\footnotetext{
${ }^{20}$ For simplicity we assume that the support is symmetric, but the result is clearly not driven by this assumption.
} 


$$
\begin{aligned}
& s_{i}(\mu)=\mu \text { for i } 2 f 1 ; 2 g \\
& { }^{1}\left(s_{1} ; s_{2}\right)\left(s_{1}\right)=1 \\
& { }^{1}\left(s_{1} ; s_{2}\right)\left(\frac{s_{1}+s_{2}}{2}\right)=1 \\
& { }^{1}\left(s_{1} ; s_{2}\right)(i W)=1 \quad s_{1}>s_{2} \text { and } s_{1}=2 x_{2} i W \\
& { }^{1}\left(s_{1} ; s_{2}\right)(W)=1 \quad s_{1}>s_{2} \text { and } s_{1}<2 x_{2} \text { i W } \\
& y\left(s_{1} ; s_{2}\right)=i^{1}\left(s_{1} ; s_{2}\right)
\end{aligned}
$$

The equilibria constructed in the proof of Proposition 1 and the one displayed above are just theoretical possibilities and we do not claim that any of them is plausible. For example, notice that in the equilibrium above, if $s_{1}>s_{2}$ and both are near zero, the policy maker believes that the state is extreme ( $\mathrm{W}$ or $\mathrm{i} W$ ): this does not seem plausible but it is necessary in order to discourage deviations, since the policy maker can not determine which expert has deviated. For this reason we argue in favor of a re- nement that eliminates these equilibria. The existence of a fully revealing equilibrium in the previous Proposition relies on the fact that following an out-of-equilibrium pair of messages we are able to construct ad hoc beliefs that support the desired outcome. The assumption that the support of $\mu$ is bounded is a radical way of restricting out of equilibrium beliefs: since no state is larger than W, clearly no out of equilibrium belief can put weight on states larger than W. The a priori assumption that the support is bounded, however, is not a good assumption, and it is not necessary for restricting out-of-equilibrium beliefs. There is not a widely accepted way to re ne beliefs in games with a continuum of types. We introduce a simple re nement on out-of-equilibrium beliefs that parallels consistency in the Sequential Equilibrium concept and has a straightforward interpretation.

We de ne an " $\mathrm{i}$ perturbed game as the game described above in which each Expert $\mathrm{i}$ independently observes the true state of nature with probability $1_{i}{ }{ }_{i}$ and with probability ${ }_{i}$ observes a random state $\beta$ : a random variable with continuous distribution $G_{i}(\phi$, density $g_{i}(\phi$ and the same support as $\mu$. We may interpret this as a situation in which each expert may commit a mistake with probability " $i$ or with this probability he is not an expert. In an "-perturbed game, the policy maker has well de- ned beliefs following any pair of messages $\mu ; \mu^{0}$, for any prior $f(\mu)$, beliefs depend on " $=(" 1 ; " 2), G(\Phi)=\left(G_{1}\left(\phi ; G_{2}(\phi)\right.\right.$ and the Experts strategies: ${ }^{1}\left(G ; " n ; s^{\natural}(\mu)\right)$ : An equilibrium is "-stable if there exists a pair of distributions $G_{i}\left(\phi\right.$ for $i=1 ; 2$ and a sequence "n $=\left(" n_{1}^{n} ;{ }^{n}\right)$ converging to zero such that out-of-equilibrium beliefs of the equilibrium are the limit as "n ! 0 of the beliefs that the equilibrium strategies would induce in an "n-perturbed game: i.e. ${ }^{1}\left(G ; " n ; S^{\natural}(\mu)\right) !{ }^{1 \ltimes}\left(S^{\natural}(\mu)\right)$. The idea behind this restriction on beliefs is very simple. In equilibrium, after a pair of messages that are inconsistent, the policy maker believes that at least one of the Experts has made a mistake. Given the distribution of the state 
of nature and the distribution of the wrong observation, the policy maker will assign a posterior probability to the event that each Expert has observed the wrong variable and with this posterior he accesses a belief on the state of nature. We require that there exist a $G_{i}(\phi i=1 ; 2$ and a sequence "n such that this process may be rationalized: this requirement imposes consistency on the construction of the posterior beliefs. As we said, it is not possible to generalize the concept of Sequential Equilibrium to cases with a continuum of agents. However, our restriction of out-of-equilibrium beliefs parallels the consistency requirement in Sequential Equilibrium. In order to have consistency in Sequential Equilibrium, in fact, we perturb strategies in order to have well de ned beliefs and we require out-of-equilibrium beliefs to be the limit of these. The perturbation of the model that we introduce may be seen in this way: each Expert i follows the equilibrium strategy with probability $1_{i}{ }_{i}$ and with probability ${ }_{i}$ follows a mixed strategy with density equal to the distribution of the wrong observation, $G_{i}(\phi$. We require beliefs to be consistent with this perturbation as " ! 0 : Note that we are not making a speci ${ }^{-} \mathrm{c}$ assumption regarding the distribution of the wrong observation in order to preserve as much generality as possible to the restriction in believes.

Given this de nition, we have:

Proposition 2 If $d=1$ and both $x_{1}$ and $x_{2}$ are large enough in absolute value, then there exists no " $\mathrm{i}$ stable fully revealing equilibrium for any W $2(i 1 ;+1)$.

Proof. In the appendix

Before moving on to the two dimensional case, it is useful to summarize the results of this section:

1) In a more general environment than Gilligan and K rehbiel [1989] it is possible to construct fully revealing equilibria (f.r.e.) with just two experts and one dimension. We have found a simple necessary and su \pm cient condition for existence of a f.r.e.

2) Even when they exist, these f.r.e. are not plausible because, at least for $x_{1}$ and $x_{2}$ large, they rely on an ad hoc construction of out of equilibrium beliefs and therefore do not survive a simple re nement.

The aim of the following section is to show that, once we consider the problem in two dimensions, the result drastically changes. The change is qualitative and yields interesting intuitions on the process of transmission of information. 


\subsection{Equilibrium in two dimensions}

\subsubsection{Simultaneous referrals}

The intuition behind the main result of this section is easily understood by considering a particular case. See Figure 2: in this case each expert has preferences that are perfectly aligned with the policy maker in one and only one dimension. Expert 2's ideal point, for example, lies in the $x$ axis so he has the same preferences as the policy maker with respect to the y dimension. However, both experts prefer point $A$ to $(0 ; 0)$ : Consider the strategies: expert i tells the truth on both dimensions; the policy maker believes each Expert on the dimension on which their preferences are aligned. For example, assume $A$ is the true state of world: the policy maker will believe that the $x$ coordinate of $A$ is equal to the $x$ coordinate of the declaration of Expert 1 ; and that the $y$ coordinate of $A$ is equal to the y coordinate of the declaration of Expert 2.

$W$ ith symmetric information, the policy maker would choose $A^{0}$ in order to achieve $(0 ; 0)$. Consider the decision of Expert 1 . Given that Expert 2 tells the truth the outcome will be on the $x$ axis; in particular ${ }^{21}$ :

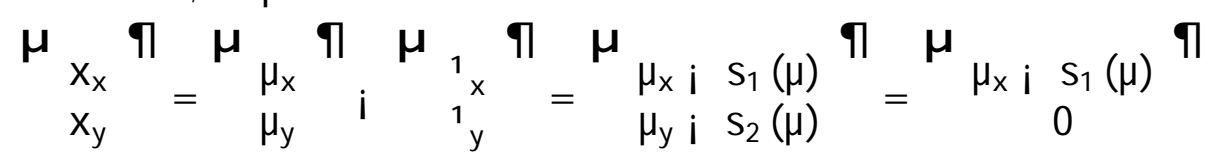

But then the optimal choice for Expert 1 is to be honest!: given Expert 2's strategy, Expert 1 knows that his message will induce an outcome on the $x$ axis; given the assumption of this example (Expert 1's ideal point on the y coordinate, quadratic utilities) the optimal point on the $x$ axis for Expert 1 is the origin. The same holds for Expert 2 and clearly the strategy is optimal for the policy maker who obtains for any $\mu$ his ideal point. Note that $A$ is a pareto improvement for both Experts but, they can not achieve it in equilibrium. Suppose that the two experts can communicate before the message are sent and, therefore, potentially can collude: this, however, would not be an equilibrium. If Expert 2 lies and reports zero the outcome, as a function of the strategy of $1 s_{1}(\mu)$ would be $x=\mu_{i}{ }^{1}=\left(\mu_{x} i s_{1}(\mu) ; \mu_{y}\right)$ (a point on the dotted line in Figure 2 ): but then the optimal choice of 1 is $s_{1}(\mu)=\mu_{x}$ (point $B$ in ${ }^{-}$gure 2 ) and the action of 2 would not be optimal.

As P roposition 3 shows, this argument may be generalized for the case where the ideal points of the agents are linearly independent.

Proposition 3 If $d=2$; then for any $x_{1}$ and $x_{2}$ such that $x_{1} \in{ }^{\circledR} x_{2} 8 \circledast 2<$, there exists a fully revealing, "-stable equilibrium.

\footnotetext{
${ }^{21}$ In this example, $s_{1}(\mu)$ is the $x$ coordinate reported by Expert $1, s_{2}(\mu)$ is the y coordinate reported by Expert 2.
} 


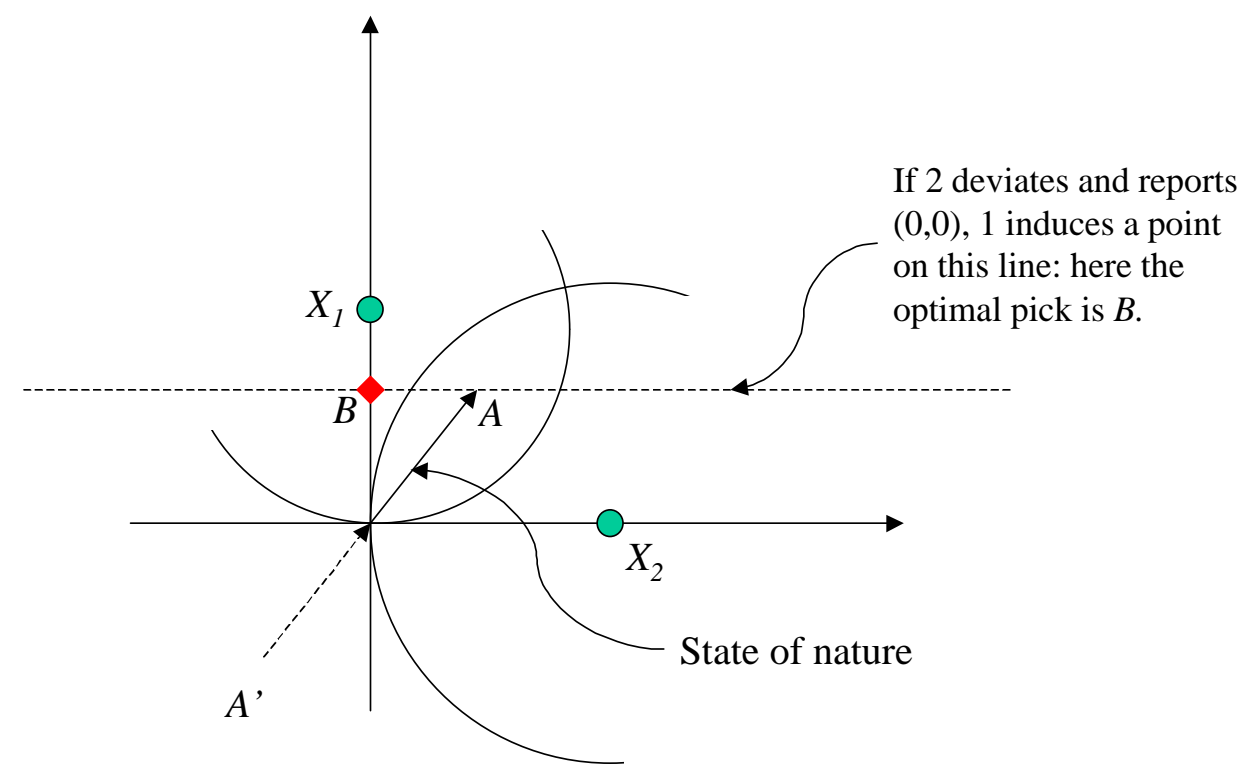

Figure 2: A special case where ideal points are on orthogonal axes. Each expert's preferences are aligned with the policy maker's on one and only one dimension.

P roof. First, some de ${ }^{-}$nitions and a useful claim. $8 a 2<; 8 i=1 ; 2$ de $^{-}$ne:

$$
I_{i}(a), \stackrel{\left(C^{\prime}\right.}{z} 2<<^{2} ; r u_{i}(0 ; 0) \phi z=a^{a}
$$

The locus $I_{i}(a)$ has a simple geometric interpretation (see Figure 3 ): $I_{i}(0)$ is the tangent of the indi ßerence curve of the ith agent at the ideal point of the policy maker; for any a $2<; l_{i}(a)$ identi ${ }^{-}$es one and only one line parallel to $I_{i}(0)$ : Notice that, given the assumption that @® $2<$ such that $x_{1}={ } x_{2} ; 8 a_{1} 2<; 8 a_{2} 2<; I_{1}(0)$ and $I_{2}(0)$ are linearly independent vector spaces ${ }^{22}$ : therefore $8 \mu 2<2$; there exists a unique vector $\left(a_{1}, a_{2}\right) 2<$ such that:

$$
\mu=I_{1}\left(a_{1}\right) \backslash I_{2}\left(a_{2}\right)
$$

We may de ${ }^{-}$ne the function $a(\mu):<^{2} !<^{2}$ that, for each $\mu$, associates the couple $a_{1}(\mu) ; a_{2}(\mu)$ uniquely de- ned by $(2)$.

It is routine to prove:

Claim 1 8(a; b; c; d) $2<$ :

\footnotetext{
${ }^{22} 8 \circledast 2 I_{1}(\mu) ; 8^{-} 2 I_{2}\left(a_{2}\right)$ then ${ }^{\circ}$ and ${ }^{-}$are linealy independent.
} 


$$
I_{i}(a) \backslash I_{j}(b)+I_{i}(c) \backslash I_{j}(d)=I_{i}(a+c) \backslash I_{j}(b+d)
$$

We are now ready to prove the proposition. Each expert is required to report a number, $\mathrm{s}_{\mathrm{i}}^{23}$. Consider the following strategies and beliefs:

$$
\begin{gathered}
s_{i}(\mu)=a_{j}(\mu) \quad 8 i ; j=1 ; 2 \text { i } 6 j \\
{ }^{1}\left(s_{1}(\mu) ; s_{2}(\mu)\right)=I_{1}\left(s_{2}(\mu)\right) \backslash I_{2}\left(s_{1}(\mu)\right) \\
y\left(s_{1}(\mu) ; s_{2}(\mu)\right)=i^{1}\left(s_{1}(\mu) ; s_{2}(\mu)\right)
\end{gathered}
$$

We claim that these strategies and this belief are an " $\mathrm{i}$ stable equilibrium. Given the other players' strategies, player $\mathrm{i}$, choosing $\mathrm{m}$; may induce a point:

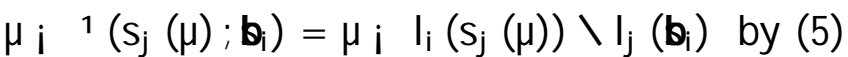

$$
\begin{aligned}
& =\mu_{\mathrm{i}} \mathrm{I}_{\mathrm{i}}\left(\mathrm{a}_{\mathrm{i}}(\mu)\right) \backslash \mathrm{I}_{\mathrm{j}}\left(\mathrm{Q}_{\mathrm{i}}\right) \text { by }(4) \\
& =I_{i}\left(a_{i}(\mu)\right) \backslash I_{j}\left(a_{j}(\mu)\right) i I_{i}\left(a_{i}(\mu)\right) \backslash I_{j}(\text { G }) \text { by de nition of } a(\mu) \\
& =l_{i}(0) \backslash l_{j}\left(a_{j}(\mu) i \text { G) by claim } 1\right. \text {. }
\end{aligned}
$$

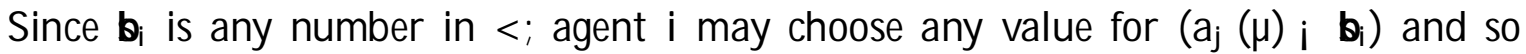
any point in $I_{i}(0)$ : But, by construction, $u_{i}$ has a unique point of tangency with $I_{i}(0)$ : the origin, i.e. the ideal point of the policy maker. The origin is the optimal outcome that $\mathrm{i}$ may induce, so the optimal strategy is to set $a_{\mathrm{j}}(\mu)$, as prescribed by the equilibrium. Therefore, there is no pro table deviation for agent $\mathrm{i}, 8 \mathrm{i}=1 ; 2$ : Clearly beliefs are consistent and the policy choice is optimal given the beliefs. The requirements for ${ }^{2}$-stability follow since there is no out of equilibrium message pair and therefore believes are always well de-ned.

The key point in understanding the general case is that if $x_{1}$ and $x_{2}$ are linearly independent, we can construct two axes that span the policy space and exploit the con ${ }^{\circ}$ ict of interest between the two experts exactly in the same way as in the particular case described above. See Figure 3: $x_{1}$ and $x_{2}$ are generic l.i. vectors in $<2$ : Given quadratic utilities, the tangents at $(0 ; 0)$ of the respective utilities are l.i.: so they span. Note that if agent $\mathrm{i}$ had to choose an outcome in $\mathrm{I}_{\mathrm{i}}(0)$ he would choose $(0 ; 0)$; i.e. the ideal point of

\footnotetext{
${ }^{23} \mathrm{Clearly}$, we can construct an equilibrium in which each agent is required to report any sequence of numbers and the policy maker ignores all of them except one. The case in which each expert is required to report a couple of numbers is particularly interesting since it may seem natural: we may interpret it as each agent being required to report the $\backslash$ coordinates" of $\mu$ : In the equilibrium that we will construct, the number that is reported will be a truthful coordinate of $\mu$; since the other coordinate is ignored, one could equivalently construct an equilibrium in which each agent truthfully reports both coordinates.
} 


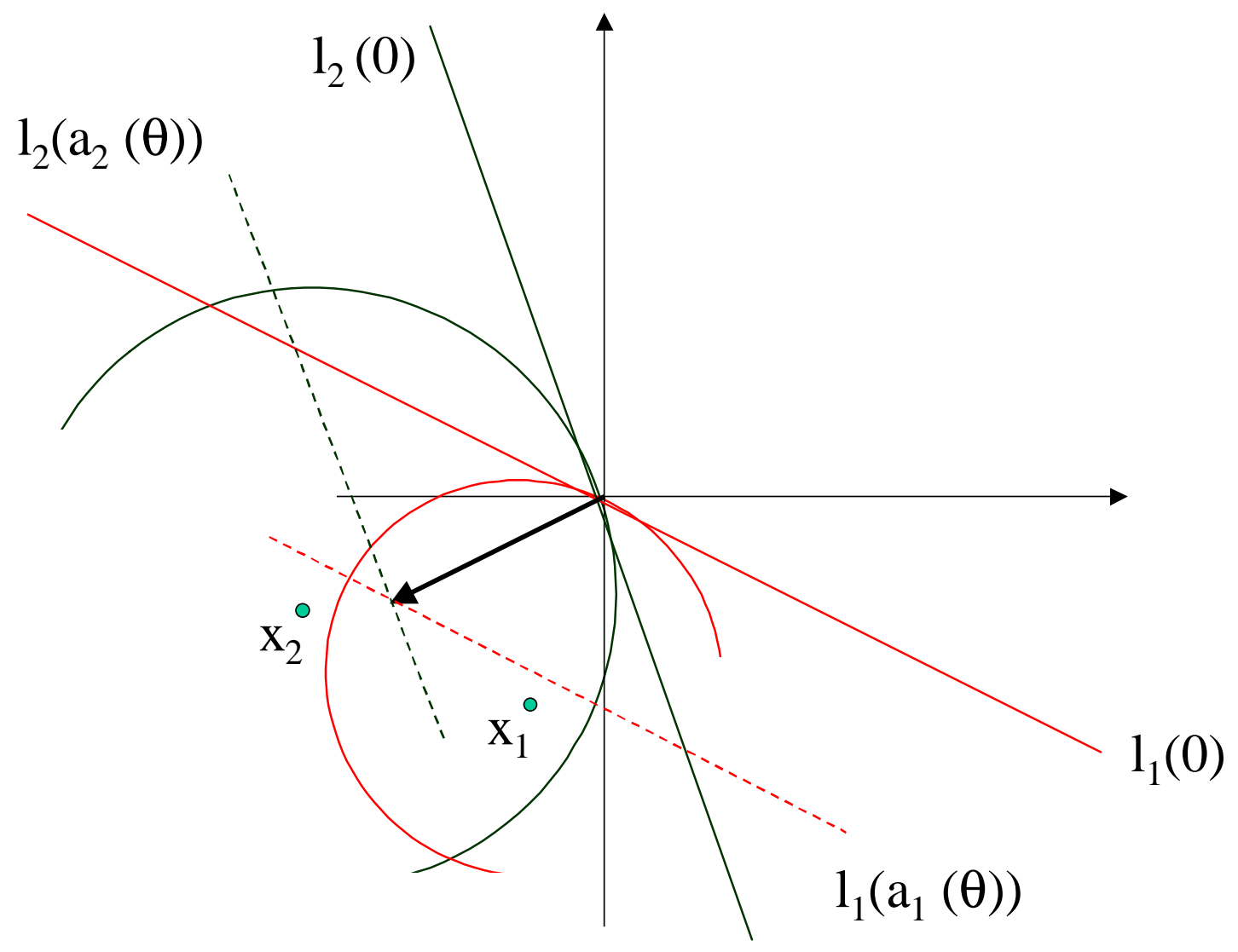

Figure 3: The general case: it is possible to construct a new coordinate system to exploit experts con ${ }^{\circ}$ ict of interest.

the policy maker; but in equilibrium this is exactly what is going to happen. Agent $\mathrm{j}$ in fact will be honest on the $l_{j}$ dimension so $i$ is forced to choose in $l_{i}(0)$ :

Notice that if $x_{1}$ and $x_{2}$ are linearly dependent, then this equilibrium is not possible: in this case $I_{1}$ and $I_{2}$ would coincide and so they would not span the entire space. However, if $x_{1}$ and $x_{2}$ are just an "linearly independent (for example $x_{1}={ }^{\circledR} x_{2}+",{ }^{\circledR} 2<$ ) then the result holds. This shows that the multi-dimensional analysis is qualitatively very di ßerent from the uni-dimensional.

A few characteristics of this equilibrium seem important. The ${ }^{-}$rst is that, as in the special case described above, the equilibrium is collusion proof. This seems a very important property: to remain in the case of two informed lobbies and a policy maker (the example in the introduction that motivated the paper) the possibility of secret agreements 
between lobbies is more than plausible and it is not desirable to rule it out. Note that this property of the equilibrium is far from being obvious. For example, the equilibrium that $\mathrm{K}$ rishna and M organ [1999a] "nd in one dimension with like biases is not collusion proof. Assume that both experts prefer a higher action than the policy maker: in K rishna and Morgan [1999a] the policy maker believes that the state is the largest of the messages sent, so no expert has a strictly preferred deviation if the other reports the truth; if, however, experts meet before sending the messages, they may decide to send messages with a negative bias: this would be an equilibrium since none of them has anything to lose from this type of coordination.

We may formalize this point. In particular we want to formalize the concept of collusion proofness of an equilibrium. For any equilibrium $f y^{\natural}\left(\phi \Phi ; S_{1}^{a}\left(\phi ; S_{2}^{\natural}\left(\phi ;{ }^{x}(\phi)\right.\right.\right.$ g we may de $^{-}$ne the induced game $\mathrm{i}\left(\mathrm{y}^{\mathbb{a}}\right)$ as a game where the players are the two experts, strategies are the same as before, and utilities are de ned: $\theta_{i}\left(s_{1} ; s_{2}\right)=u_{i}\left(\mu+y^{\not}\left(s_{1} ; s_{2}\right)\right)$ : Clearly the original equilibrium is an equilibrium of this game, but there may be other equilibria. A ssume that there exists an equilibrium that pareto dominates $s_{1}^{a}(\mu) ; s_{2}^{a}(\mu)^{24}$ : If we assume that agents may communicate before playing the game, then the original game would be at least suspect: agents would coordinate on the pareto superior equilibrium. Therefore we may de`ne:

De $e^{-}$nition 2 An equilibrium of the original expertise game is collusion proof if the induced game $\mathrm{i}\left(\mathrm{y}^{\mathbb{a}}\right)$ has no pareto superior equilibria.

This de ${ }^{-}$nition does not coincide with de nitions used in other work on collusion ${ }^{25}$. In this literature, collusion is ruled out if players can not coordinate on an action that it is strictly pareto superior. In this sense the re- nement is inspired by Aumann's Strong Nash Equilibrium concept. In order to pursue this approach, however, it is necessary to assume that players can sign binding agreements at the colluding stage ${ }^{26}$ : this seems a strong assumption. The approach followed in this paper, on the contrary, is in line with the Coalition Proofness concept introduced by Bernheim, Peleg and Whinston [1987]. The structure of the model and the role of players allow us to restrict attention to the coalition formed only by Experts. What is important, is that the coalition of Experts can not commit to a join strategy. This is consistent with the structure of the model because it preserves its non cooperative nature.

Using De- nition 2, we have:

\footnotetext{
${ }^{24} \mathrm{At}$ least one agent is strictly better and both are not worse $0{ }^{\circledR}$.

${ }^{25}$ See for instance Tirole [1992] or B aliga [1999].

${ }^{26}$ It is not necessarily true, in fact, that a pro- le of strategies that yields the pareto improvement is a nash equilibrium.
} 
Proposition 4 Given that utilities are quadratic and $x_{1} 6{ }^{\circledR} x_{2} 8 \circledast 2<$, the equilibrium constructed in Proposition 3 is collusion proof.

Proof. Assume it is not. Then there exists a strictly pareto superior equilibrium $s_{1}^{0}(\mu) ; s_{2}^{0}(\mu)$; which clearly must induce an outcome di ßerent from the origin. The following condition must hold, otherwise there would be a pro ${ }^{-}$table deviation for one of the experts:

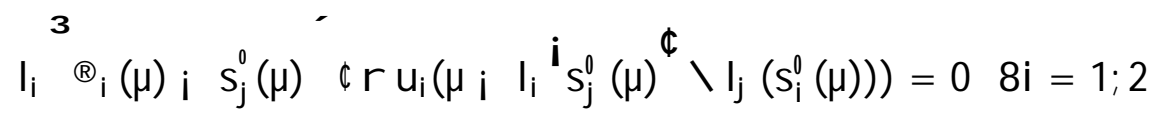

The ${ }^{-}$rst term of the LHS of (7) is the direction of allowed deviation for agent $\mathrm{i}$ at equilibrium, the second is the gradient of $i$ 's utility at the equilibrium outcome. Condition (7) means that for each agent, at the equilibrium outcome, the indi ßerence curve of the agent must be tangent to the direction of allowed deviation for the agent, if this is not true then there is a pro ${ }^{-}$table deviation. Given quadratic utilities, the locus of possible outcomes with this property is the line connecting the ideal point of agent $i$ with the origin: however the intersection of these two loci contains only the origin since $x_{i} \in \mathbb{B} x_{j}$.

Proposition 5 actually proves more than is required for $\mathrm{De}^{-}$nition 2 : in $\mathrm{i}\left(\mathrm{y}^{\mathrm{a}}\right)$ there is a unique equilibrium. However, while the results presented before hold for any strictly concave utility of the Experts, this result is not general and depends on the assumption of quadratic utility. The result may be generalized to other utility functions that are Iregular", but it is easy to see that we may " nd examples of strictly concave utilities for which it fails to hold.

The second observation is that the result is robust to changes in the information structure. One change is particularly important and, in some sense, is the opposite case of collusion. In the model we assume that the experts' ideal points are common knowledge; let's now assume that each expert does not know the ideal point of the other, but that the policy maker knows both of them: the equilibrium is robust to this change. This also is a very important property: it is plausible to assume that the policy maker knows both the experts (he may have chosen them...); however there is no reason to assume that experts know each other perfectly. In the equilibrium described above, each expert knows that his message induces a point on the tangent line of his own indi ßerence curve at the policy maker's ideal point. He does not need to know completely the other expert's utility function; he just needs to know that the policy maker is informed: in equilibrium the policy maker Ineutralizes" the other expert's bias and forces a choice on his tangent line. Notice, however, that the gradient of both utility functions at the ideal point of the policy maker must be common knowledge in order to construct the new \coordinate system" used in the equilibrium. This is an interesting point: it is not in the interest of 
the policy maker that experts ignore each other characteristics: the policy maker wants them to be aware of their con ${ }^{\circ}$ ict of interest.

The most important characteristic of this equilibrium, however, is that it yields insights into the information transmission process that can not be appreciated in the analysis of the one-dimensional case. The "rst and most important has been mentioned, and was commented in the introduction: the importance of the local behavior of the experts utilities at the policy maker's ideal point. In one dimension, clearly there is only one direction of increasing of utility. In two dimensions, the issue of determining in which direction utility increases most is very important and is a key variable in any equilibrium. Using information on gradients, it is possible to exploit the experts' con ${ }^{\circ}$ ict of interest in the optimal way. The tangent of the experts' indi ßerence curve at the policy maker's ideal point is important because we know it is the only direction of \movement" in which the utility of the expert decreases for any deviant message. Forcing the expert to induce a point in this locus is a crucial ingredient of the equilibrium.

A related point, which, as we discussed in the introduction, has important implications for the informational theory of legislative organizations, is that the proximity of ideal points is not important for information transmission. In the equilibrium that we constructed, for example, distance is not important at all: if ideal points are linearly independent, then even if they are arbitrarily distant, we can construct a fully revealing equilibrium.

Last, but not least, the analysis of the two-dimensional case yields useful insights also into the study of the $\backslash$ open vs. closed" rule question. In equilibrium each agent $i$ is given complete power to decide the policy outcome on the $I_{i}$ dimension, so we may say that the equilibrium resembles a closed rule. However, this would be only super ${ }^{-}$cially true. In the one-dimensional case the policy choice is trivially irrelevant; in the two dimensional case the dimensions of choice are endogenous, part of the equilibrium. It is true that $i$ has the choice on the ith dimension, but this dimension was chosen by the policy maker in the optimal way: $i$ is free to choose the outcome on that dimension, but in equilibrium he is forced to be honest! The analysis of the multidimensional case, therefore, revisits the notion of 'open' and 'closed' rule and questions the relevance of this distinction.

We conclude this section with two somewhat more technical remarks:

1) In the model, we use quadratic utilities for simplicity: it is not di \pm cult to see that the results hold under the general assumption of concave utility functions. The condition $\mathrm{x}_{1} \in \mathbb{R}_{2} 8 \circledast$ is necessary here because we have assumed quadratic utilities, in general, if we assume concave utilities, it is not necessary: we need $r u_{i}(0 ; 0) ; r u_{j}(0 ; 0)$ to be linearly independent. If this condition holds, in fact, we can construct a new coordinate system exactly in the same way as in Proposition 3. 
2) If utility functions are not di ßerentiable (for example, Leontiev utilities) but are concave, then the results still hold: in this case we have a multiplicity of equilibria that, exactly in the same way as Proposition 3, achieve a fully revealing equilibrium.

3) In the previous section we analyzed the case with two Experts and two dimensions. However two Experts are su \pm cient for full revelation also in more then two dimensions. Consider the three dimensional case $^{27}$. Consider Expert $i\left(i=1\right.$ or 2 ) and ${ }^{-} x$ the plane that is tangent to his indi ßerence curve at the policy maker's ideal point, call this plane $T_{i}$ : For Expert $1,{ }^{-} x$ one vector on $T_{1}$; call it $v^{1}$ : For Expert $2,^{-} x$ two linearly independent vectors in $T_{2}$, call them $v_{1}^{2}, v_{2}^{2}$ Clearly $f v^{1} ; v_{1}^{2} ; v_{2}^{2} g$ span the three-dimensional space. Consider an equilibrium in which the declaration of Expert1 is interpreted as a coordinate in the $v^{1}$ dimension; the declaration of Expert $2^{28}$ is interpreted as the coordinate in the $v_{1}^{2}, v_{2}^{2}$ dimension. Given that Expert 1 reports the truth, Expert 2 will have to choose a point on $T_{2}$; by construction the optimal choice in $T_{2}$ is the ideal point of the policy maker, so Expert 2 will reveal the true coordinate (in the $v_{1}^{2}, v_{2}^{2}$ coordinate system); given that Expert 2 is honest, Expert 1 will have to choose a point in $v_{1}$ and therefore is honest. This argument can be generalized to four dimensions and two experts.

\subsubsection{Sequential referrals}

When experts report simultaneously the condition required by the equilibrium constructed in Proposition 3 is not su \pm cient to guarantee the existence of a fully revealing equilibrium.

To see what may go wrong in the argument made in the previous section when experts report sequentially consider Figure 4. Assume that the state of the world is $\mu$ (the thick arrow). Consider the following deviation for Expert 1, the ${ }^{-}$rst to report: instead of reporting $a_{2}(\mu)$, he reports $s_{1}(\mu)=a_{2}(\mu) ;$ " as the $I_{2}$ coordinate. If Expert 2 observe this choice, Expert 1 will know that Expert 2 will choose a point in the locus that have coordinate $I_{2}$ equal to $a_{2}(\mu) ;\left(a_{2}(\mu) ; "\right)="$ in the $I_{1} ; I_{2}$ coordinate system. But now the optimal choice in this locus is point $M$, which is preferred by both Experts to the origin. Therefore Proposition 3 does not necessarily hold if experts report sequentially.

As we said in the introduction, K rishna and Morgan [1999a] have proven that when experts report sequentially and there is only one dimension, no fully revealing equilibrium exists: it is natural to ask whether in a multidimensional environment there is a su \pm cient condition for the existence of a fully revealing equilibrium.

The reason why in the example of $\mathrm{Fig} \mathrm{4,} \mathrm{there} \mathrm{are} \mathrm{problems} \mathrm{is} \mathrm{that} \mathrm{Expert} \mathrm{1,} \mathrm{given} \mathrm{the}$ policy maker's strategy, knows that if he gives the opportunity to Expert 2 to determine an outcome $M$ that also Expert 2 prefers, then also Expert 2 will lie. Given the reaction

\footnotetext{
${ }^{27}$ I thank David A usten Smith for this observation.

${ }^{28} \mathrm{~T}$ herefore Expert 2 is in ${ }^{\circ}$ uential in two dimensions.
} 


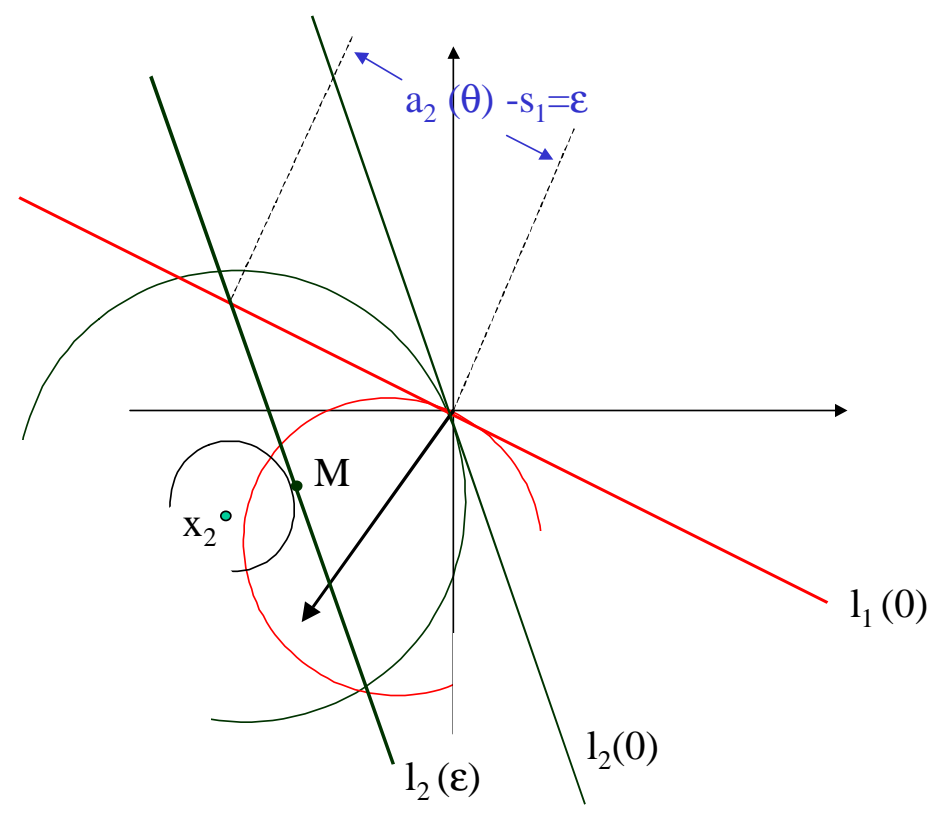

Figure 4: Sequential referrals: example of a pro ${ }^{-}$table deviation.

function of Expert 2, we may ${ }^{-}$nd the points that Expert 1 may induce, i.e. the points that given the message of Expert 1 the optimal message of Expert 2 would determine. If among these points there is a point that 1 prefers to 0 then we have a pro ${ }^{-}$table deviation and no more a fully revealing equilibrium. To ${ }^{-}$nd a su \pm cient condition for a fully revealing equilibrium we have to ${ }^{-} \mathrm{nd}$ a condition that guarantees that 0 is the best point for Expert 1 among the points that he may induce. This turns out to be very simple.

Proposition 5 If $d=2$, then for any $x_{1}$ and $x_{2}$ such that $x_{1} \in \mathbb{B} x_{2} 8 \circledR$ and $r u_{1}(0 ; 0)$ a $r u_{2}(0 ; 0)=0$ there exists a fully revealing, " $i$ stable equilibrium.

P roof. Consider the same strategies of Proposition 1. Expert 1 can induce any point $x={ }^{-} x_{2}$ : i.e. any point that is a linear combination of $x_{2}$ and the origin. In fact, 8a $2<$ chosen by Expert 1 , Expert 2 will choose a point such that $I_{2}(a)$ is tangent to the indi ßerence curve: for any choice of a the locus of such points is ${ }^{-} x_{2} 8^{-} 2<$. So by choosing a, Expert 1 can induce any point on this locus. However since $r u_{1}(0 ; 0)$ $r u_{2}(0 ; 0)=0$. the origin is the point that expert 1 prefers among the ones that he may induce.

The intuition for Proposition 6 may be seen in Figure 5. If the direction of Expert 1's highest increase of utility is not orthogonal to the one of Expert 2 (in the picture $r u_{i}(0 ; 0)$ form an obtuse angle) there is a point that Expert 1 can induce and that is preferred to 


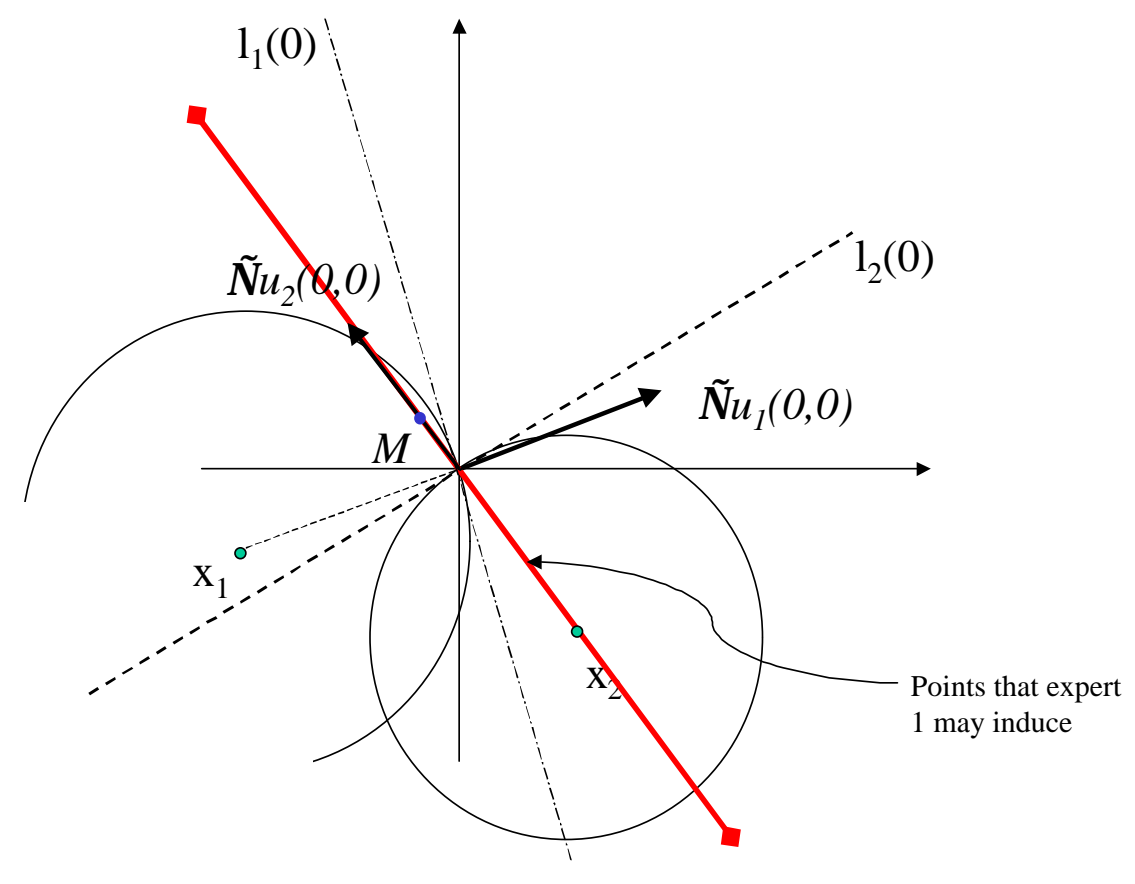

Figure 5: Illustration of the su \pm cient condition for existence of a fully revealing equilibrium with sequential referrals

0 : if the gradients, instead, are orthogonal, the point that Expert 1 prefers among the ones that he may induce is 0 , the policy maker's ideal point.

\section{Conclusions}

In this paper we have shown that there are important insights to be gained from the analysis of multidimensional cheap talk: it is not just a technical change in the model, the results are qualitatively di ßerent. Contrary to the onedimensional case, full revelation of information is typically possible in two dimensions (or more) and the equilibrium that supports this outcome has very good properties: it is robust to perturbations of the model such as errors in Experts' information, sequential reports or collusion. Clearly full revelation is due to some simplifying assumptions and should not be expected literally in real life; however we believe the results are important for at least two reasons:

1) The assumptions made are the same, mutatis mutandis, to the ones made in models in the one-dimension.

2) The model sheds light on the mechanics of information transmission and yields insight into, and a new interpretation of, the information transmission process. 
In particular, this second point is important. We discussed the implication of this theory in the \informational" theories vs. I distributional" theories debate and we have seen that the theory presented here seems to explain an empirical puzzle: in particular, we argued that the fact that the loutlier principle" is not clearly supported by empirical evidence is not su \pm cient to reject informational theories of legislative organization (IT LO). Our argument, on the other hand, is not su \pm cient to reject distributional theories in favor of IT LO either: new evidence that takes in consideration the multidimensionality of problems is needed. This work suggests new empirical tests. In particular, more than the absolute \distance" of ideal points, empirical studies should look for Icomplementaries" in experts' preferences: experts that have distant ideal points with respect to the policy maker, but also have considerable con ${ }^{\circ}$ icts of interests among themselves along di ßerent dimensions of problems, may reveal information. These con ${ }^{\circ}$ icts of interest in di ßerent dimensions (complementaries) could be detected if they are relevant for information transmission.

The paper has not considered many further interesting questions: probably the most important is endogeneity of information acquisition by Experts. The design of an optimal organization should reward Experts for the e®ort they put in the information acquisition. This may force the policy maker to limit his own ability to extract information. One way to do this is to separate jurisdictions in the policy space, giving autonomy to di ßerent policy makers who control distinct dimensions of the problem: this may lead to imperfect information transmission and grant some residual rent to Experts. This extension is left for further research. 


\section{A ppendix}

\subsection{Proof of lemma 1}

Step 1. (there is a truthful fully revealing equilibrium) Assume that ${ }^{1}\left(s_{1}(\mu) ; s_{2}(\mu)\right)$; $y\left(s_{1}(\mu) ; s_{2}(\mu)\right) ; s_{i}(\mu)$ for i $2 f 1 ; 2 g$ is a fully revealing equilibrium. De- ne $e\left(x^{0} ; x\right)=$ ${ }^{1}\left(s_{1}\left(x q ; s_{2}(x)\right)\right.$ and $\left(x^{0} ; x\right)=y\left(s_{1}\left(x 9 ; s_{2}(x)\right)\right.$. Then $e(g ; s) ; s=\mu$ for i $2 f 1 ; 2 g$,

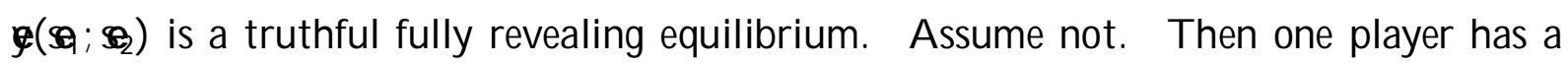
strictly preferred deviation. Assume expert i prefers to report $\mu^{0}$ in state $\mu:$ If $s_{1}(\mu 9)=$ $s_{1}(\mu)$ then this generates the same belief and the same policy maker's action: so it can not be strictly preferred. If $s_{1}(\mu 9) \in s_{1}(\mu)$ then the deviation was available also in the previous equilibrium, but this is a contradiction. A similar argument can be applied to the policy maker.

Step 2. (degenerate out of equilibrium beliefs) For any equilibrium with out of equilibrium belief ${ }^{1}\left(s_{1}(\mu) ; s_{2}(\mu)\right)(\mu)$ the optimal choice of the policy maker is $y\left(\mu^{0} ; \mu\right)=$ i $E_{1}\left(s_{1}(\mu) ; s_{2}(\mu)\right)(\mu)$ and if the out of equilibrium beliefs are non degenerate, then there is an outcome equivalent equilibrium with degenerate beliefs, i.e. that assign probability one on one value of $\mu$ : Given the belief ${ }^{1}$, in fact, consider the expected utility of the policy maker $\mathrm{Eu}(\mu ; \mathrm{y})=\mathrm{i}_{\mathrm{i}=1}\left(\mu_{\mathrm{i}} \mathrm{i} \mathrm{y}_{\mathrm{i}}\right)^{21}(\mu) \mathrm{d} \mu$; where the subscript indicates the coordinate and the policy maker's ideal point is zero; we may re-write it:

$$
\begin{aligned}
& i_{i}^{P=1}\left(\mu_{i} i y_{i}\right)^{21}(\mu) d \mu \\
& =i_{P}^{i=1}\left(y_{i}^{2}+\mu_{i}^{2} i 2 y_{i} \mu_{i}\right)^{1}(\mu) d \mu=i{ }_{i=1}^{P}{ }_{i}^{2}\left(y_{i}^{2}+E_{1}\left(\mu_{i}\right)^{2} i 2 y_{i} E_{1}\left(\mu_{i}\right)\right)^{1}(\mu) d \mu+K \\
& =i \quad \sum_{i=1}^{2}\left(E_{1}\left(\mu_{i}\right) i y_{i}\right)^{2}+K
\end{aligned}
$$

where $K$ is a constant that does not depend on $y_{i}$ : The optimal choice, therefore, is $y\left(\mu^{0}, \mu\right)=i E_{1}(\mu)$ : Since the optimal choice depends only on $E_{1}(\mu)$; we may introduce an outcome equivalent degenerate belief function:

$$
1\left(\mu^{0}, \mu\right)=\begin{array}{lr}
1 / 2 & 1 \text { if } \mu=E_{1}(\mu) \\
0 & \text { else }
\end{array}
$$

\subsection{Proof of proposition 1}

Necessary condition. We prove that for $W, j x_{1} j+j x_{2} j$ we may ${ }^{-}$nd a truthful fully revealing equilibrium. Without loss of generality assume $x_{2}>0$ and $x_{1}<0$ : For any couple $\mu^{0}, \mu$ an equilibrium speci ${ }^{-}$es a posterior belief for the policy maker on the distribution of $\mu_{;}{ }^{1}\left(\mu^{0} ; \mu\right)$. In a truthful fully revealing equilibrium ${ }^{1}(\mu ; \mu)(\mu)=1$; i.e. the belief distribution is degenerate and assigns probability 1 to state $\mu$ : Given an out of equilibrium couple $\mu^{0} ; \mu$ the posterior distribution is not necessarily degenerate. However, it su \pm ces to prove that there is a fully revealing equilibrium with out of equilibrium belief 
that assign positive probability only on one point. W ith a slight abuse of notation call this point ${ }^{1}\left(\mu^{0}, \mu\right)$ : i.e. given $\mu^{0}, \mu$; the policy maker will believe that the true state is ${ }^{1}\left(\mu^{0}, \mu\right)$ with probability one.

For any couple $\mu^{0} ; \mu$ we only have to ${ }^{-} n d^{1}\left(\mu^{0} ; \mu\right)$ such that:

$$
\begin{array}{ll}
u_{1}\left(\mu i^{1}\left(\mu^{0}, \mu\right)\right) & u_{1}(0) \\
u_{2}\left(\mu^{0} i^{1}\left(\mu^{0} ; \mu\right)\right) & u_{2}(0)
\end{array}
$$

The interpretation of (9) and (10) is the following. If the true state is $\mu$, then $\mu_{\mathrm{i}}{ }^{1}\left(\mu^{0}, \mu\right)$ is the outcome if expert 1 deviates and declares $\mu^{0}$ : (9) just requires that for any state of the world $\mu$; any deviation from the equilibrium is not strictly pro table for 1 . This condition is not enough for an equilibrium; we also need that ${ }^{1}\left(\mu^{0}, \mu\right)$ is such that in state $\mu^{0}$ expert 2 doesn't want to deviate: given, in fact, that the couple $\left(\mu^{0}, \mu\right)$ induces the belief ${ }^{1}\left(\mu^{0}, \mu\right)$ we want to rule out the case in which in state $\mu^{0}$ experts 2 deviates reporting $\mu$; this is the reason we impose also the other inequality. Inequality (9) implies that one of the following two inequalities is satis ${ }^{-}$ed:

$$
\begin{array}{lll}
\text { a1 } \mu_{\mathrm{i}}{ }^{1}\left(\mu^{0} ; \mu\right), & 0){ }^{1}\left(\mu^{0} ; \mu\right) \quad \mu \\
\text { a2 } \mu_{\mathrm{i}}{ }^{1}\left(\mu^{0} ; \mu\right) & \left.2 x_{1}\right) & { }^{1}\left(\mu^{0} ; \mu\right), \mu_{\mathrm{i}} 2 \mathrm{x}_{1}
\end{array}
$$

and the other condition implies that one of the following two equations is satis ${ }^{-}$ed:

b1 ${ }^{1}\left(\mu^{0} ; \mu\right), \mu^{0}$

b2 ${ }^{1}\left(\mu^{0} ; \mu\right) \quad \mu^{0}$ i $2 x_{2}$

There exist an equilibrium if for all the out of equilibrium couples we can ${ }^{-} n d a^{1}\left(\mu^{0} ; \mu\right)$ such that one inequality of the ${ }^{-}$rst group and one of the second are simultaneously satis ${ }^{-}$ed and ${ }^{1}\left(\mu^{0}, \mu\right) 2[; \mathrm{W} ; \mathrm{W}]$ : i.e. there is no incentive to deviate and the belief is in the support of $\mu$ : We consider the possible cases and we show that the set of beliefs that satisfy the required conditions is non empty for any couple $\left(\mu^{0}, \mu\right)$ :

Case 1 . If $\mu^{0} \quad \mu$ then we may satisfy al and b1 choosing ${ }^{1}\left(\mu^{0}, \mu\right) 2\left[\mu^{0}, \mu\right]$ :

Case 2. Case 2.1: $\mu^{0}>\mu ; \mu, 0$ : Consider b2 and a1, so ${ }^{1} \quad \min \left(\mu^{0} i 2 x_{2} ; \mu\right)$. If $\mu_{i}^{0} 2 x_{2}$, i W just take ${ }^{1}\left(\mu^{0}, \mu\right) 2\left[i \mathrm{~W} ; \min \left(\mu^{0} ; 2 x_{2} ; \mu\right)\right.$ ]: it is possiblesince $\left[i \mathrm{~W} ; \min \left(\mu^{0} \mathrm{i}\right.\right.$ $\left.2 x_{2} ; \mu\right)$ ] would be non empty. If $\mu^{0} i 2 x_{2}<i W$ then we have that:

$$
\mu^{0}<2 x_{2} \text { i W and } j 2 x_{2} j>W \text { so } j x_{1} j<j x_{2} j
$$

The ${ }^{-}$rst inequality follows by simple manipulation; the second follows by the fact that $\mu^{0}$, 0 so $2 x_{2}>W$ and by $W, j x_{1} j+j x_{2} j$ we have $\left.j 2 x_{2} j>W, j x_{1} j+j x_{2} j\right) \quad j x_{1} j<j x_{2} j$

Consider then $a 2$ and b1. Inequality a2 requires ${ }^{1}\left(\mu^{0}, \mu\right), \mu_{i} 2 x_{1}$ which is implied by ${ }^{1}\left(\mu^{0} ; \mu\right), \mu^{0} \mathrm{i} 2 \mathrm{x}_{1}$ since $\mu^{0}>\mu$ which is implied by ${ }^{1}\left(\mu^{0} ; \mu\right), 2 x_{2}$ i W i $2 x_{1}$ because of the ${ }^{-}$rst inequality of (11). By the assumption $W, j x_{1} j+j x_{2} j$ we have $2 x_{2} i W i$ 
$2 \mathrm{x}_{1} \quad \mathrm{~W}$ so the set $\left[2 \mathrm{x}_{2} \mathrm{i} \mathrm{W} ; 2 \mathrm{x}_{1} ; \mathrm{W}\right]$ is not empty and it is just su \pm cient to take $12\left[2 x_{2} ; W\right.$ i $\left.2 x_{1} ; W\right]$ :

Case $2.2 \mu^{0}>\mu ; \mu<0$ : Case 2.2.1 $\mu^{0}>\mu ; \mu<0 ; \mu^{0}, 0$; by al and b2 it is su \pm cient $1 \quad \min \left(; W_{;} ; \mu^{0} ; 2 x_{2}\right)$ : if $\mu^{0} ; 2 x_{2}$, i $W$ choose $^{1}\left(\mu^{0} ; \mu\right)=$ i W: If $\mu^{0} ; 2 x_{2}<$ i W then 11 holds. Consider $a 2$ and b1 which are satis ${ }^{-}$ed if ${ }^{1}, \max \left(W ; \mu_{i} 2 x_{1}\right)$ but $\mu<0$ and, by the second inequality of $11, j 2 x_{1} j<W$ so just choose ${ }^{1}=W$ :

Case 2.2.2. $\mu^{0}>\mu ; \mu<0 ; \mu^{0}<0$ : Inequalities b2 and a1 are satis ${ }^{-}$ed if 1 $\min \left(; W ; \mu^{0} \mathbf{i} 2 x_{2}\right)$ : if $\mu^{0} i 2 x_{2}, i W$ choose ${ }^{1}\left(\mu^{0}, \mu\right)=i W$. If $\mu^{0} i 2 x_{2}<i W$ then the ${ }^{-}$rst inequality of (11) holds. Then consider a2 and b1 which are implied by ${ }^{1}>$ $\max \left(\mu^{0}, \mu_{i} 2 x_{1}\right)$ which is implied by ${ }^{1}>\mu^{0}{ }_{i} 2 x_{1}$ since $\mu<\mu^{0}$, which is implied by ${ }^{1}\left(\mu^{0}, \mu\right), 2 x_{2} ; W ; 2 x_{1}$. As in case 2.1 , by the assumption $W, j x_{1} j+j x_{2} j$ we have $2 x_{2} ; \quad W ; 2 x_{1} \quad W$ so the set $\left[2 x_{2} ; W ; 2 x_{1} ; W\right]$ is not empty and it is just su \pm cient to take ${ }^{1} 2\left[2 x_{2}\right.$ i W i $\left.2 x_{1} ; W\right]$ :

Therefore for any deviation we can ${ }^{-}$nd the required out of equilibrium belief such that the deviation is not strictly pro table.

Su \pm cient condition: we prove that if $\mathrm{W}<\mathrm{jx} \mathrm{x}_{1} \mathrm{j}+\mathrm{jx_{2 }} \mathrm{j}$ then there can not exist a fully revealing equilibrium. By lemma 1 it su \pm ces to show that no truthful fully revealing equilibrium with degenerate out of equilibrium beliefs exists. For this we just need to prove that there exist $a \mu$ and $a \mu^{0}$ such that no couple of inequality $a$ and $b$ can be satis" ed. Consider $\mu^{0}=\operatorname{minf} 2 x_{2} i W_{i} " ; W ;$ "gand $\mu=\mu^{0}{ }^{\prime}$ " for " $>0$ arbitrarily small.

Since $\mu^{0}<2 x_{2}$ i W condition b2 never holds. Condition b2, in fact, would require ${ }^{1}\left(\mu^{0}, \mu\right) \quad \mu^{0}$ i $\left.2 x_{2}<i W\right) \quad{ }^{1}\left(\mu^{0}, \mu\right)<$ i W which is not possible since ${ }^{1}\left(\mu^{0}, \mu\right)$ must belong to the support of $\mu$ : If b2 does not hold the possible couple of inequalities that can be satis ${ }^{-}$ed are $a 1, b 1$ and $a 2, b 1$. Inequalities $a 1$ and b1 clearly never hold together. Consider a2. If $2 x_{2} i W_{i} "<W_{i}$ " then

1. $\mu$ i $2 x_{1}=2 x_{2}$ i W i 2" i $2 x_{1}>2 x_{2}$ i $2^{\prime \prime}$ i $2 x_{1}$ i $x_{2}+x_{1}=x_{2}$ i $x_{1}$ i $2^{\prime \prime}>W$

since " is arbitrarily small and by assumption $W<j x_{1} j+j x_{2} j$ :

If $2 x_{2} i W_{i} ", W_{i}$ " then

$$
\text { 1. } \mu_{\mathrm{i}} 2 \mathrm{x}_{1}=\mathrm{W} \text { i } 2^{\prime \prime} \text { i } 2 \mathrm{x}_{1}>\mathrm{W}
$$

since $\mathrm{x}_{1}<0$ and $\mathrm{j} " \mathrm{j}<\mathrm{jx} \mathrm{j}$ : In either case we have a contradiction and so also inequality a2 can not hold. 


\subsection{Proof of proposition 2}

Once we introduce ${ }^{2}$-stability, Lemma 1 does not necessarily hold. In the proof of Lemma 1 , in fact, we have exploited the indeterminacy of out of equilibrium beliefs, but now ${ }^{2}$ stability restricts the set of feasible out of equilibria beliefs. To see that we may have a f.r.e. that is not truthful, consider this case: each expert is pooling, and the declaration of each expert reveals that the state is in a set (say $A_{i}$ for agent $i$ ); however the intersection of the two sets is a singleton and, so, information is fully revealed. With "i stability the out-of-equilibrium beliefs depend on the equilibrium strategies (i.e. on the sets $A_{i}$ ). In particular, it may be possible that, through choice of the sets $A_{i}$, we may construct out-of-equilibrium beliefs that support full revelation: such an equilibrium would clearly have beliefs that are di @erent from the case of truthful strategies. Therefore, in order to prove that there exists no ${ }^{2}$-stable f.r.e., it is not enough to prove that there exists no ${ }^{2}$-stable truthful fully revealing equilibrium. For this reason, in the following proof we do not invoke Lemma 1.

Assume that there exists a fully revealing eqpilibrium. For any message $s_{i}$ sent in equilibrium by agent $i$ de ne a set: $A_{i}\left(s_{i}\right):=\quad \beta 2 f^{-} s_{i}(\beta)=s_{i}$. If there exists $a$ fully revealing equilibrium, it must be that for any $\mu, A_{1}\left(s_{1}(\mu)\right) \backslash A_{2}\left(s_{2}(\mu)\right)=f \mu g$ : T wo conditions must be satis ${ }^{-}$ed.

1. If there exist a f.r.e. and one agent pools, then the other agent will be able to choose any point in the pooling set: otherwise some state would never be revealed. I 1 particular, for any $\beta$ in $A_{i}\left(s_{i}\right)$; there must be a message $s_{j}$ such that $A_{1}\left(s_{i}\right) \backslash A_{2}\left(s_{j}\right)=\quad$ R :

2. To have incentive compatibility, it is necessary that agent $j$ doesn't strictly prefer any point $\mu^{0}$ in $A_{i}\left(s_{i}(\mu)\right)$ to $\mu$ : For this reason, it must be that for any $t_{1+1} ; t_{1} 2 A_{1}\left(s_{1}\right)$ such that $t_{1+1}>t_{1}$, then $t_{1+1}, t_{1}+2 x_{2}$. A ssume not: if $t_{1+1}<t_{1}+2 x_{2}$; then in state $t_{1+1}$ agent 2 may report that the state is $t_{1}$ so the outcome would be $0<t_{1+1} ; t_{1}<2 x_{2}$ : this would be a pro table deviation for agent 2 . In the same way, for $v_{n+1} ; v_{n} 2 A_{2}(\mu)$ such that $v_{n+1}<v_{n}$; it must be $v_{n+1} \quad v_{n}+2 x_{1}$ :

We now consider the beliefs that may follow after a pair of out-of equilibrium signals $s_{1}\left(\mu^{9}\right) ; s_{2}\left(\mu^{9}\right)$ if "-stability is satis" ed; we then prove that given these beliefs we always have a pro" table deviation for at least one Expert.

For each I $2 A_{i}(\mu)$; the posterior probability that the state is I given the true state

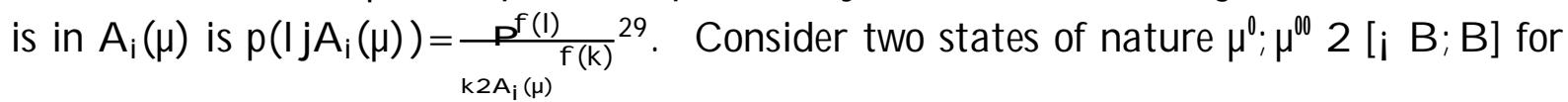

\footnotetext{
${ }^{29} \mathrm{As}$ an heuristic justi ${ }^{-}$cation for this consider $A_{2}\left(\mu ; \#=f\left[I_{1} ; I_{1}+\# ;\left[I_{2} ; I_{2}+\# ;:: g\right.\right.\right.$ so, by Bayes' rule,

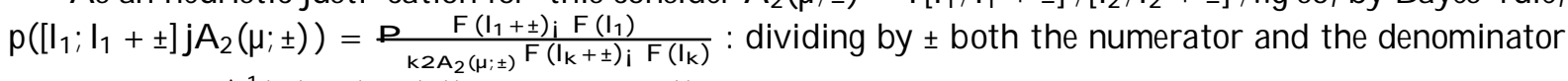

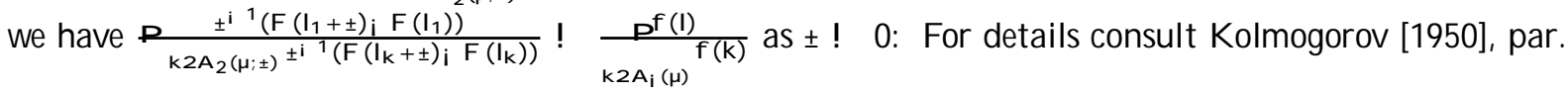


$B<\min f j x_{1} j ; j x_{2} j g$ and $\mu^{0} \sigma \mu^{\oplus}$. For Expert 1 ; consider the set $A_{1}\left(s_{1}\left(\mu^{9}\right)\right.$ so that, by the choice of $B, A_{1}\left(s_{1}\left(\mu^{9}\right) n f \mu^{0} g z[i B ; B]\right.$; in the same way, for Expert 2, consider the set $A_{2}\left(s_{2}\left(\mu^{0}\right)\right)$ : as before $A_{2}\left(s_{2}\left(\mu^{0}\right) n f \mu^{\infty} g z[\dot{\phi} B ; B]\right.$. We can write:

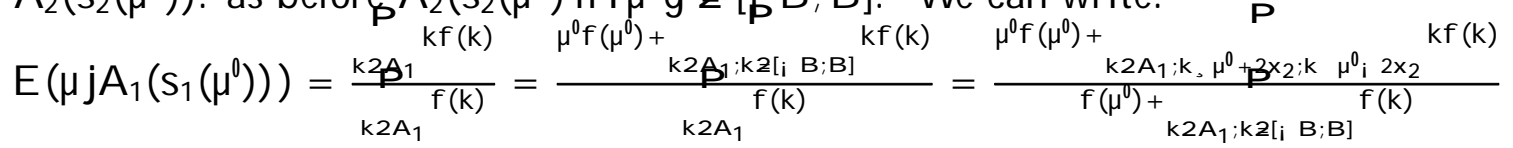

Since, by the existence of the ${ }^{-}$rst moment of the distribution, the second term of the numerator of the last expression converges to zero as $\mathrm{x}_{2}$ converges to 1 ; it must be that, for $x_{2}$ large enough, $E\left(\mu j A_{1}\left(s_{1}(\mu 9) 2\left[i B^{Q}\right]\right.\right.$ where $2\left(B ; \operatorname{minfjx}_{1} j ; j x_{2} j g\right)$. In the

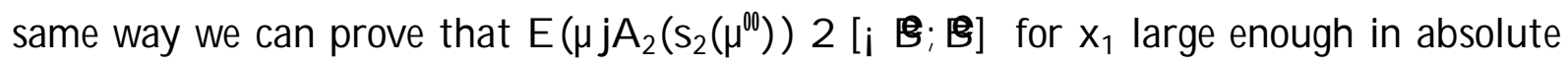
value. Given the equilibrium strategies, in any " $\mathrm{n}$-perturbed game the event $\backslash$ observation of the couple $s_{1}\left(\mu 9 ; s_{2}\left(\mu^{9}\right)\right.$ by the policy maker" is the union of three disjoint events:

a : event in which agent 1 is right and agent 2 observes the wrong state;

b: event in which agent 2 is right and agent 2 observes the wrong state;

$c$ : event in which both agents observes the wrong state.

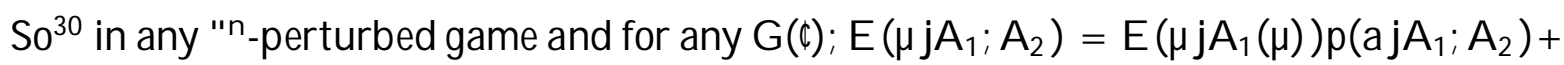
$E\left(\mu j A_{2}(\mu)\right) p\left(b j A_{1} ; A_{2}\right)+E(\mu) p\left(c j A_{1} ; A_{2}\right)$ : Therefore $E\left(\mu j A_{1} ; A_{2}\right) 2$ [i $\left.B ; B\right]$ for min $f j x_{1} j ; j x_{2} j g$ large enough. It follows that, after a pair $s_{1}\left(\mu 9 ; s_{2}\left(\mu^{\mathbb{Q}}\right)\right.$, beliefs must be in $[; \mathbb{B} ; \mathbb{B}$ ] as

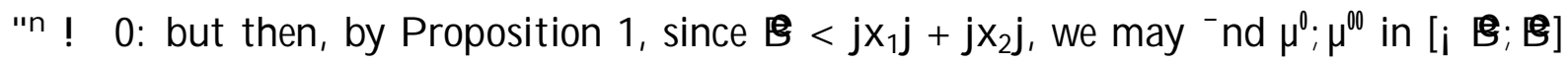
such that either Expert 1 has a pro ${ }^{-}$table deviation in state $\mu^{\infty}$ or Expert 2 has a profitable deviation in state $\mu^{0}$. Since this holds for any distribution of the wrong signal for the experts $G(\phi$ and any converging sequence " $n$, it follows that no out-of-equilibrium belief that satisfy the requirement of " $i$ stability supports a f.r.e.

3 , page 51 .

${ }^{30}$ The probabilities $p\left(k j A_{1} ; A_{2}\right)$ for $k=a ; b ; c$ are constructed with Bayes' rule following the logic described in note 25: they will depend on $f(\phi)$ and $g(\phi)$ and the sequence "n. It is not necessary to specify them since we only need them to be in $[0 ; 1]$. 


\section{R eferences}

[1] Austen-Smith, David, and William Riker 1987. \A symmetric information and the coherence of legislation". American Political Science Review, 81:897-918.

[2] Austen Smith, David, 1991. \Information acquisition and orthogonal argument", in Political economy: institutions competition and representation, proceedings of the seventh international symposium in economic theory and econometrics, eds. Barnet, William A., M elvin Hinich J . and Scho- eld Norman J .

[3] Austen Smith, David, IInterested experts and Policy Advice: Multiple Referrals under Open Rule", Games and Economic Behavior, 1993, pp.:3-44.

[4] Austen-Smith David, Banks J e®rey, 1999. Positive Political Theory: Collective Preferences. Ann Arbor: University of Michigan Press.

[5] Baliga Sandeep, 1999. \M onitoring and Collusion with \Soft" Information". J ournal of Law Economics and Organization; 15(2). p.434-440.

[6] Bernheim B. Douglas, Peleg Bezalel, Whinston Michael D., 1987. J ournal of Economic Theory. Vol.42. No.1. p.1-12

[7] Crawford P. Vincent, Sobel J oel, 1982. \Strategic Information Transmission, Econometrica, Vol. 50, No. 6: 1431-1451.

[8] Diermeier Daniel, Feddersen T imothy J ., 1998. \Information and Congressional Hearings, mimeo.

[9] Epstein David, 1998. \Partisan and Bipartisan Signalling in Congress", J ournal of Law, Economics \& Organization, v.14, n2: 183-204.

[10] Farrell J oseph and Gibbons R obert, 1986. "Cheap Talk with two Audiences", American Economic Review. Vol.79. No. 5. p. 1214-1223.

[11] Gibbons, Robert. 1986. \Learning Equilibrium Models of A rbitration", American Economic Review. Vol.78. No. 5. p. 896-912.

[12] Gilligan, Thomas W., K rehbiel, K eith. 1987. \Collective Decision-M aking and Standing Committees: An informational Rational for Restrictive Amendments Procedures", J ournal of Law, E conomics \& Organization, 3: 287-335. 
[13] Gilligan, Thomas W ., K rehbiel, K eith. 1989. \A symmetric Information and Legislative R ules with a Heterogeneous Committee" , A merican J ournal of Political Science, Vol 33. No. 2

[14] K olmogorov A.N., 1950. Foundations of the theory of probability, N ew Y ork. Chelsea Publishing Company.

[15] K rehbiel, K eith, 1991. Information and legislative Organization. A nn A rbor. University of Michigan Press.

[16] K rishna Vijay, M organ J ohn, 1999a. \A model of Expertise". Mimeo.

[17] K rishna Vijay, M organ J ohn, 1999b. \A symmetric Information and legislative Rules: Some A mendments". Mimeo

[18] M ilgrom Paul and R oberts J ohn, 1986. \R elying on information of interested parties", Rand J ournal of Economics. Vol 17. No. 1, p.18-32.

[19] Plott, CR.,1966. \A notion of equilibrium and its possibility under majority rule". American Economic review, 57:787-806.

[20] Laver, Michael, Shepsle Kenneth A.(eds.), ICabinet Ministers and Parliamentary Government", Cambridge University Press 1994.

[21] Tirole J ean, 1992. ICollusion and the theory of organization", in Advances in Economic Theory: Six World Congress. Volume 2. E conometric Society Monographs, No 21, Ed. J ean-J acques Laßont. p. 151-20 